\title{
The Long Non-Coding RNA-I4327.I Promotes Migration and Invasion Potential of Endometrial Carcinoma Cells by Stabilizing the Potassium Channel Kca3.I
}

This article was published in the following Dove Press journal: OncoTargets and Therapy

\author{
Yingli Zhang \\ Ping Zhang \\ Lu Chen \\ Lingqin Zhao \\ Jianqing Zhu (ID \\ Tao Zhu (D)
}

Department of Gynecologic Oncology, Zhejiang Cancer Hospital, Hangzhou 310022, People's Republic of China
Correspondence: Tao Zhu; jianqing Zhu Department of Gynecologic Oncology, Zhejiang Cancer Hospital, I Banshan East Road, Hangzhou 310022, People's Republic of China

Email zhutao@zjcc.org.cn;

zhujq@zjcc.org.cn
Background: The intermediate-conductance $\mathrm{Ca}^{2+}$-activated potassium channel (Kca3.1) plays a key role in maintaining intracellular $\mathrm{Ca}^{2+}$ homeostasis and is involved with the carcinogenesis of many human tumors including endometrial carcinoma. However, the underlying mechanism is still remained to be further elucidated.

Methods: The relationship between Kca3.1 and the clinicopathological characteristics of endometrial carcinoma was analyzed using UALCAN cancer database, and its expression was determined by immunohistochemistry. The Kca3.1 binding candidate lncRNAs were screened using RNA immunoprecipitation sequencing assay in the endometrial carcinoma cell line. MTT assay and transwell assay were used to confirm the cell proliferation migration and invasion, respectively. FACS was used to determine the cell cycle distribution. The overexpression efficiency of the lncRNAs was detected by qRT-PCR. The expression of EMT related proteins and the stability of Kca3.1 were analyzed by Western blot assay.

Results: Kca3.1 is related to clinicopathological characteristics of endometrial carcinoma, such as tumor stages. Several Kca3.1 binding lncRNAs were obtained from RNA immunoprecipitation sequencing assay. Stable expression of lncRNA-14327.1, one of the candidate lncRNAs, led to significant upregulation of Kca3.1 protein level, cell migration and invasion abilities, but suppressed cell proliferation and induced cell cycle arrest. Additionally, our data also demonstrated that Lenti-lncRNA-14327.1 could stabilize the protein of Kca3.1 and subsequently increase intracellular $\mathrm{Ca}^{2+}$ concentration. Transfection of siRNA-Kca3.1 significantly inhibited cell migration and invasion, and attenuated the EMT in Lenti-lncRNA-14327.1 stably expressed endometrial carcinoma cells.

Conclusion: Taken together, our results demonstrated that the lncRNA-14327.1 promoted cell migration and invasion potential of endometrial carcinoma cells by stabilizing Kca3.1 protein, implying that the lncRNA-14327.1/Kca3.1 might be a promising therapeutic target in endometrial carcinoma, particularly the metastatic one.

Keywords: endometrial carcinoma Kca3.1, lncRNA-14327.1, metastasis, EMT

\section{Introduction}

Endometrial carcinoma is the most common malignancy of the female genital tract and is a growing number of in incidence, especially a high incidence rate in China. ${ }^{1,2}$ The mortality of endometrial carcinoma is only inferior to ovarian and cervical cancers and is presently increasing annually. ${ }^{3}$ In recent years, The Cancer 
Genome Atlas (TCGA) database issued a comprehensive analytical report of genome, transcriptome, and proteome profiles in 373 patients diagnosed with endometrial carcinoma. ${ }^{4}$ Additionally, this alliance decided four prognostic categories (from good to poor) for classification of endometrial carcinoma: (I) polymerase $\varepsilon$ (POLE) hypermutation; (II) microsatellite instability (MSI) ultramutation; (III) lower copy number and (IV) higher copy number. However, in spite of advancements on early detection and better treatment options in the last few years, palindromia and metastasis can still occur leading to poor prognosis..$^{5}$ Therefore, it is desirable to further elucidate the molecular mechanisms that mediate endometrial carcinoma progression, especially metastasis.

As the most various classes of ion channels, the potassium channel can regulate numerous cellular functions in both stressful and non-stressful cells. Furthermore, due to its cell surface approachability, the potassium channel offers great therapeutic potential in many human diseases, including cancers. ${ }^{6}$ The intermediate conductance $\mathrm{Ca}^{2+}$ activated potassium channel Kca3.1, also known as SK4 or IK (encoded by the KCNN4 gene), is indispensable to maintain intracellular calcium homeostasis. ${ }^{7,8}$ The abnormal expression of Kca3.1 has been demonstrated to be associated with malignancy in most kinds of human cancers, such as pancreatic cancer, kidney cancer, ovarian cancer, colorectal cancer, breast cancer, lung cancer and glioblastoma. ${ }^{9-16}$ However, the role of Kca3.1 in the regulation in endometrial carcinoma remains to be further illustrated.

Long non-coding RNAs (lncRNAs) belong to a subgroup of noncoding RNAs that are at lowest 200 nucleotides in length and without protein-coding capacity. ${ }^{17,18}$ Across the previous studies, there are mounting evidences that the abnormal expression of long non-coding RNAs (lncRNAs) plays key roles in tumorigenesis and cancer metastasis. ${ }^{19-22}$ Moreover, the expression profilings of IncRNAs may facilitate the diagnosis and prognosis of human cancer, implying that lncRNAs may serve as efficacious therapeutic targets for intervention. Previous evidence also shows that several lncRNAs are important in the pathogenesis of endometrial carcinoma, including MALAT1, HOTAIR and H19. ${ }^{23-26}$ To be better understanding of molecular-level information provided by lncRNAs, we can inform diagnosis and therapy in endometrial carcinoma.

In the present study, the expression levels of Kca3.1 were found to be significantly up-regulated in endometrial carcinoma tissues compared to normal tissues, and correlations between Kca3.1 expression and different grades, stage were obtained from the datasets of TCGA (The Cancer Genome Atlas) project. Moreover, using RNA immunoprecipitation sequencing assay, we found that Kca3.1 may be highest targeted by lncRNA-14327.1, a part of an uncharacterized noncoding RNA LOC105378179. However, whether lncRNA-14327.1 is involved in the aberrant expression of Kca3.1, and the activation of EMT in endometrial carcinoma remains undiscovered. This study aimed to explore the function of lncRNA-14327.1 on Kca3.1 regulation in endometrial carcinoma. We also investigated the relationship between lncRNA-14327.1, Kca3.1 and EMT in endometrial carcinoma, to seek a potential therapeutic target for endometrial carcinoma, particularly the metastatic one.

\section{Materials and Methods Clinical Specimens and Cell Culture}

Twenty-five endometrial carcinoma biopsy specimens were collected from patients at the Zhejiang Cancer Hospital at the time of surgery and fixed in buffered formaldehyde solution, transferred to $70 \%$ ethanol, embedded in paraffin, sectioned into 5um sections and kept in $-20^{\circ} \mathrm{C}$. All patients provided a written informed consent for the use of these clinical materials in research, and the project was approved by the Institutional Ethics Committee of Zhejiang Cancer Hospital. The human endometrial carcinoma cell line HEC-1A was purchased from The Cell Bank of Type Culture Collection of the Chinese Academy of Sciences (Shanghai, China), and cultured in DMEM medium supplemented with 10\% FBS. All cell lines were cultured in a humidified incubator in an atmosphere of $5 \%(\mathrm{v} / \mathrm{v}) \mathrm{CO}_{2}$ at $37{ }^{\circ} \mathrm{C}$.

\section{Immunohistochemistry}

Tissue slices were deparaffinage and dehydration. The activity of endogenous peroxidase was blocked with 3\% hydrogen peroxide. Heat-induced antigen retrieval (HIER) was accomplished all slices in $0.01 \mathrm{M}$ citrate buffer by using a steamer at $95{ }^{\circ} \mathrm{C}$. All primary antibodies were diluted with BSA to a concentration of 1:100 and applied to the slices. Incubation for 45 mins at room temperature followed by incubation with a DakoEnVision+System-HRP Labelled Polymer for 30 mins at room temperature. Diaminobenzidine was then applied for 10 mins. The slices were redyed with hematoxylin and then were dehydrated, covers lipped and visualized.

\section{RNA Immunoprecipitation}

The RIP assay was performed using a Thermo Fisher RIP kit (Thermo Fisher Scientific, MA, U.S.A) following the 
manufacturer's instructions. Briefly, the cell lysates in RIP lysis buffer and RNAs were incubated with the magnetic beads coupled with IgG or anti-Kca3.1 antibody at $4{ }^{\circ} \mathrm{C}$ overnight. After the RNA was purified from the RIP production, lncRNAs were analyzed by high-throughput sequencing assay and validation was performed by qPCR assay.

\section{Plasmid Construction and Transfection}

The sequences of lncRNA-14327.1, IncRNA-14324.1, and lncRNA-14327.3 were synthesized and then subcloned into the vector pcDNA3.1 (Invitrogen, CA, USA), generating the overexpression vectors for pcDNA-14327.1, pcDNA14324.1 and pcDNA-14327.3. The integrity of the respective plasmid constructs was confirmed by DNA sequencing. The plasmid formed a complex with Lipofectamine 2000 (Invitrogen) for $20 \mathrm{~min}$ at room temperature, and transfection was applied at $37^{\circ} \mathrm{C}$ for $24 \mathrm{~h}$. The empty pcDNA3.1 vector was used as a contral. The expression of the lncRNAs was then determined by qPCR.

\section{Generation of Stable Cell Lines with Overexpression of IncRNA- I4327.I}

For stably overexpressing lncRNA-14327.1, the lncRNA14327.1 sequence was subcloned into the LV-13 (pLentiEF1a-LUC-F2A-Puro-CMV) vector (GenePharma, Shanghai, China), and endometrial carcinoma cells were infected with the concentrated virus. Subsequently, cells were treated with $2 \mu \mathrm{g} / \mathrm{mL}$ puromycin for 2 weeks to select for stable cell line, in which the expression of IncRNA-14327.1 was validated by qPCR analysis.

\section{RNA Interference of Kca3.I}

Short interfering RNAs for Kca3.1 and non-targeting siRNAnegative control were obtained from GenePharma. Cells were transfected with siRNA using Lipofectamine 2000 reagent according to the manufacturer's instructions. Before any treatment, cells were incubated for $24 \mathrm{~h}$ or $48 \mathrm{~h}$ and the silence efficiency of the siRNA was determined by Western blotting.

\section{In vitro Migration and Invasion Assays}

For the migration assay, a transwell system (24 wells, 8 $\mu \mathrm{m}$ pore size with a poly-carbonate membrane, Corning) was applied according to the manufacturer's protocols. Cells were seeded into the upper chambers and cultured in serum-free DMEM medium. The lower compartment was stuffed with $10 \%$ FBS in DMEM as a chemotactic factor. After incubation for $24 \mathrm{~h}$, the cells that remained in the upper chamber were removed, and the cells at the bottom of the insert were fixed, stained in $0.5 \%$ crystal violet and counted under a microscope (Olympus Corp., Tokyo, Japan). The results were averaged over three independent experiments. For invasion assay, the invasion chambers were pre-coated with Matrigel (1:3 dilution; $\mathrm{BD}$ Biosciences) at $37^{\circ} \mathrm{C}$ for $30 \mathrm{~min}$. The following steps were described as same as migration assay.

\section{Western Blotting}

Cells were lysed in RIPA buffer containing protease inhibitor cocktail (Sigma). After separation by electrophoresis on a $10 \%$ or $12 \%$ SDS-PAGE gel, proteins were transferred onto PVDF (polyvinylidene difluoride) membranes. Then, the membranes were blocked with 5\% milk. After that, the membranes incubated with primary antibodies against Kca3.1, E-cadherin, N-cadherin, TIMP3 (all four from Abcam) at $4{ }^{\circ} \mathrm{C}$ overnight. The corresponding HRP (horseradish peroxidase)-conjugated secondary antibody was incubated for $1 \mathrm{~h}$ at room temperature. Signals were visualized after chemiluminescence reaction with the HRP substrate. $\beta$-actin or GAPDH (CST) was applied as a loading control.

\section{Cell Viability Assay}

The cell viability was assayed in 96-well plates of endometrial carcinoma cell lines using the MTT assay. In brief, the pcDNA3.1, pcDNA-14327.1, pcDNA-14324.1 or pcDNA-14327.3 transfected cells were seeded into 96well plates at a density of 5000 cells/well and allowed to incubate for $24 \mathrm{~h}$ and $48 \mathrm{~h}$. After that, the cells followed by the MTT assay to determine cell survival rates. The absorbance of each sample was read at $570 \mathrm{~nm}$ using a microplate reader. For the cell viability of stable cell lines with overexpression of lncRNA-14327.1 was the same progresses as above.

\section{Cell Cycle Analysis}

After treated, cells were harvested and washed with $1 \times$ PBS (phosphate-buffered saline) and fixed in $75 \%$ ethanol at $-20{ }^{\circ} \mathrm{C}$. The fixed cells were washed with $1 \times \mathrm{PBS}$, incubated for $30 \mathrm{~min}$ at $37^{\circ} \mathrm{C}$ with RNase A $(100 \mu \mathrm{g} / \mathrm{mL})$ and $0.1 \%$ TritonX-100, stained with propidium iodide $(30$ $\mu \mathrm{g} / \mathrm{mL}$ ), and analyzed by flow cytometry (FACS Calibur, BD Biosciences). The percentages of cells at G1, S, and G2/M were calculated by the FlowJo software program (Flowjo, LLC) based on the DNA content. 


\section{Intracellular Ca2+ Levels}

To measure intracellular $\mathrm{Ca} 2+$ levels, the Calcium Assay Kit from Abcam was used according to the manufacturer's protocols.

\section{Statistical Analysis}

All data are expressed as the means \pm S.D. from at least three independent experiments. All statistical analyses were performed using GraphPad Prism 5.0 (GraphPad Software, Inc., La Jolla, CA, USA) and SPSS 13.0 (SPSS, Inc., Chicago, IL, USA) software packages. Statistical significance was determined using two-sided Student's $t$-test, and $\mathrm{P}<0.05$ (*) was considered significant.

\section{Results}

\section{Relationship Between Kca3.I Expression} and Characteristics of Clinical Pathological in Endometrial Carcinoma

\section{Patients}

To investigate the clinical relevance of Kca3.1 in endometrial carcinoma, we first analyzed the TCGA database and found that the expression level of Kca3.1 was much higher in endometrial carcinoma tissue than in adjacent normal tissues (Figure 1A). Moreover, high Kca3.1 expression was associated with advanced tumor-node-metastasis (TNM) stage (Figure 1B).

To explore whether the Kca3.1 expression profiling in clinical specimens was consistent with the database, the Kca3.1 protein level in 25 paired normal tissues and endometrial carcinoma tissues was detected by Immunohistochemistry. These analyses revealed that the protein level of Kca3.1 was significantly upregulated in endometrial carcinoma tissues compared with the normal counterparts (Figure 1C, representative results were shown). Taking together, these results indicated that the upregulation of Kca3.1 might play a crucial role in endometrial carcinoma development and progression.

\section{The IncRNA- I 4327.I Might Directly Bind to Kca3.I to Promote Cell Migration in Endometrial Carcinoma Cells}

To determine the association between lncRNA and Kca3.1 in endometrial carcinoma, RNA immunoprecipitation (RIP) was carried out in HEC-1A cells. Using a specifically Kca3.1 targeted antibody to pull down the complex, followed by RNA seq and qPCR validation. In the Kca3.1 antibody group, the content of IncRNA ranked in the top three are presented (Table 1).

To further explore the biological role of these three lncRNAs in endometrial carcinoma, we first constructed their overexpression plasmids and examined the transfection efficiency in HEC-1A cells and observed that pcDNA14327.1, pcDNA-14324.1 and pcDNA-14327.3 were highly expressed after transfection for both $24 \mathrm{~h}$ and $48 \mathrm{~h}$ compared with the empty vector control (Figure 2A). The sequences for the primers used are listed in Table 2. Next, Western blot assay was used to analyze the protein levels of Kca3.1 in the HEC-1A cells that transfected with pcDNA-14327.1, pcDNA-14324.1, pcDNA-14327.3 and pcDNA3.1, respectively. As shown in Figure 2B, all three lncRNAs could upregulate the protein expression of Kca3.1, but the cells transfected with pcDNA-14327.1 expressed the highest level of Kca3.1. However, the results of the MTT assay revealed that the lncRNA-14327.1, lncRNA-14324.1 and lncRNA-14327.3 overexpression significantly inhibited cell proliferation (Figure 2C).

To further examine the effect on the tumor behavior of lncRNA-14327.1, lncRNA-14324.1 and lncRNA-14327.3 overexpression in endometrial carcinoma cells, migration ability was measured by the transwell assays. As shown in Figure 2D, lncRNA-14327.1 overexpressed cells migrated fastest, the lncRNA-14324.1 overexpressed cells were the second, while the IncRNA-14327.3 overexpressed cells were the last one. These data confirmed that HEC$1 \mathrm{~A}$ cells with a higher expression level of IncRNA14327.3 were more aggressive. Taken all together, these results indicated that up-regulation of $\operatorname{lncRNA}-14327.3$ increased Kca3.1 protein level might be related to the onset of endometrial carcinoma cell migration.

\section{The IncRNA- |4327.I Stabilizes Kca3.I}

\section{Protein to Increase Ca2+ Inward}

\section{Transport in Lenti-I4327.I HEC-I A Cells} To further elucidate the function of IncRNA-14327.1 in endometrial carcinoma cells in vitro, we constructed the IncRNA-14327.1 stably overexpressed HEC-1A cell line and its control using the lentivirus system, and the lncRNA-14327.1 expression level in stable HEC-1A cells was determined by qPCR assay (Figure 3A). Next, the stable HEC-1A cells were analyzed by Western blot. As expected, a significant increase in protein level of Kca3.1 in the lenti-14327.1 group compared with those observed in the lenti-control group (Figure 3B). 
A

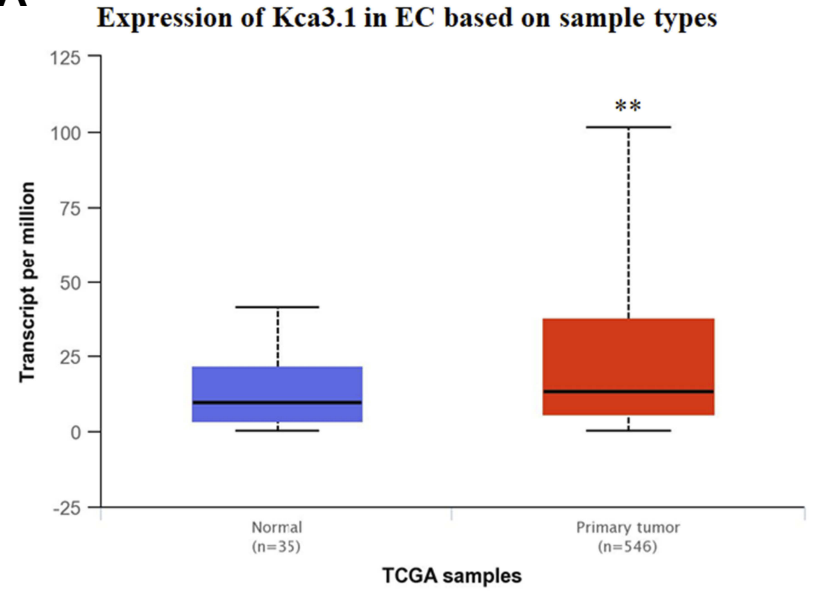

C

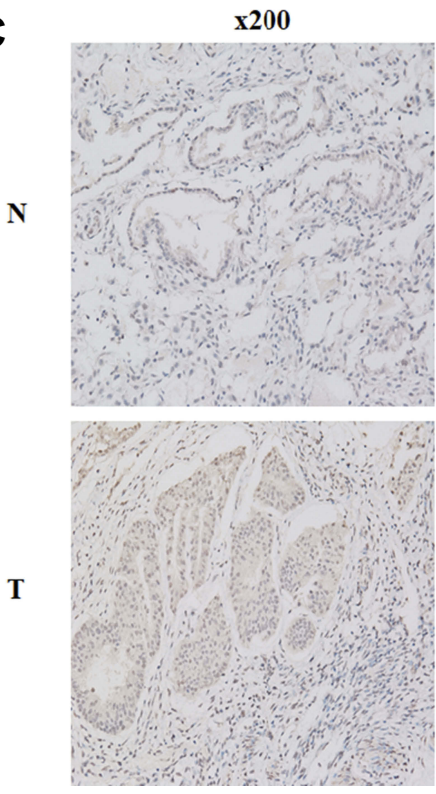

B
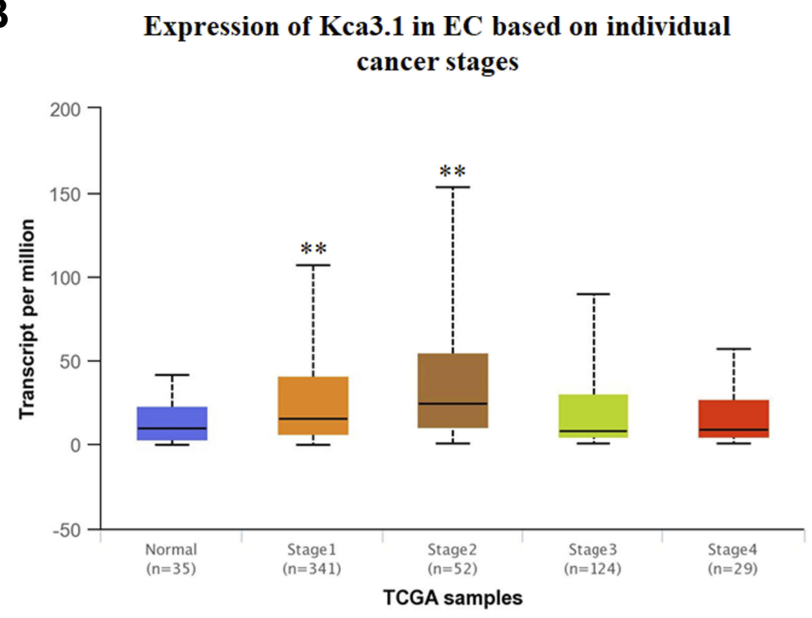

Figure I Kca3.I (KCNN4) is highly expressed in endometrial carcinoma tissues. (A) Graph showing expression of KCNN4 in the normal and primary tumors. Data were obtained from the TCGA database. (B) Graph showing expression of KCNN4 on individual cancer stages of endometrial carcinoma. Data were obtained from the TCGA database. (C) Human endometrial carcinoma tissues were stained with anti-human KCNN4 monoclonal antibodies. Brown color indicates KCNN4 protein levels, with counterstaining by hematoxylin in blue. Shown are representative images of endometrial carcinoma tissues with different positive expressions.

To confirm the effect of increasing endogenous lncRNA14327.1 expression on Kca3.1 protein degradation, we compared the half life time of Kca3.1 protein between IncRNA-14327.1 stably overexpressed HEC-1A cell line and its control. The stability of Kca3.1 protein was found to be

Table I The Result of RIP Sequencing

\begin{tabular}{|l|l|l|l|l|}
\hline t_name & Length & Control & Kca3.I & Significant \\
\hline MSTRG.I4327.I & 513 & 0 & $10,867.39$ & Yes \\
MSTRG.I4324.I & 307 & 0 & 2218.13 & Yes \\
MSTRG.I4327.2 & 305 & 0 & 588.62 & Yes \\
\hline
\end{tabular}

upregulated more than twofold after overexpression of IncRNA-14327.1 compared with the control cells in a CHX pulse-chase assay (Figure 3C). Since Kca3.1 is a regulator of intracellular $\mathrm{Ca}^{2+}$, we next tested whether upregulation of Kca3.1 following stable lenti-14327.1 expression was associated with intracellular $\mathrm{Ca}^{2+}$ homeostasis. As expected, a remarkable enhancement of intracellular concentration of $\mathrm{Ca}^{2+}$ in the lenti-14327.1 group compared with those in the lenti-control group was observed (Figure 3D). Taken together, our results suggested that the lncRNA-14327.1 could indeed stabilize Kca3.1 protein and finally increase the intracellular concentration of $\mathrm{Ca}^{2+}$ in HEC-1A cells. 


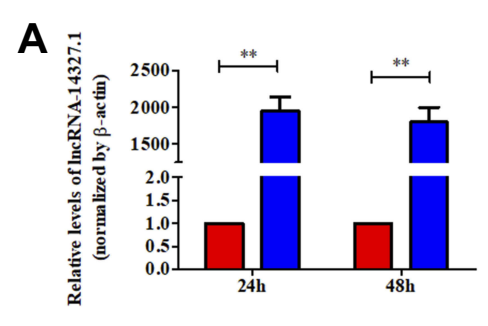

$\square$ pcDNA3.1 $\square$ IncRNA-14327.1

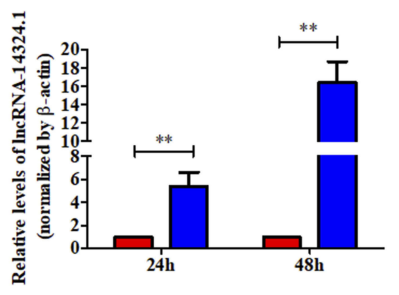

$\square$ pcDNA3.1
IncRNA-14324.1

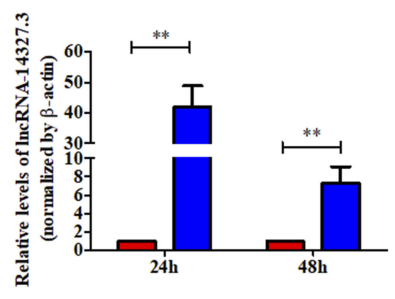

$\square$ pcDNA3.1

lncRNA-14327.3

B

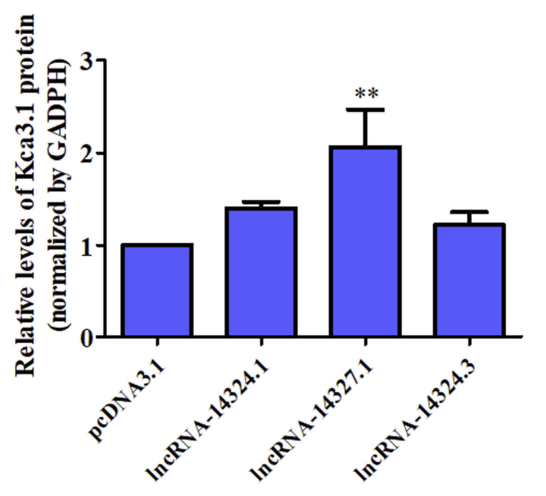

C

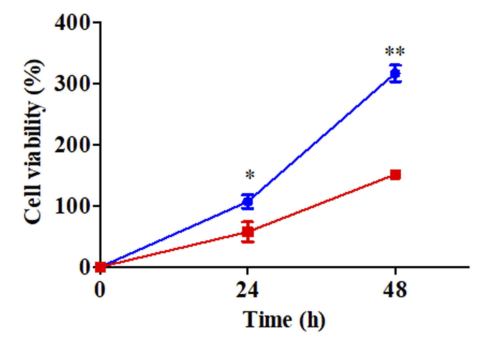

$\rightarrow$ Control $\quad \rightarrow$ IncRNA-14327.1

$45 \mathrm{kDa}$

Kca3.1

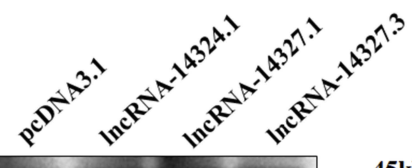

GAPDH

$36 \mathrm{kDa}$

D

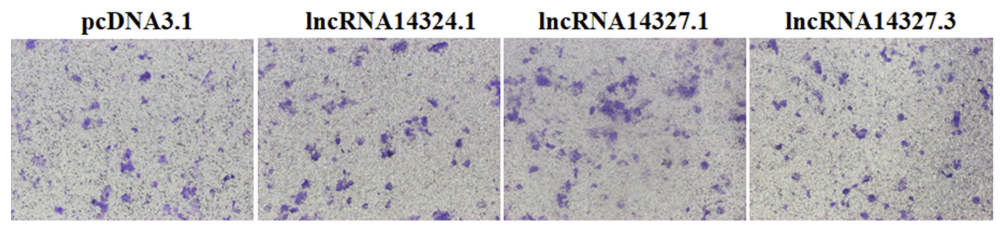

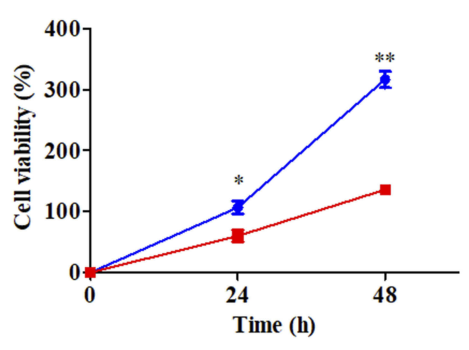

$\rightarrow$ Control

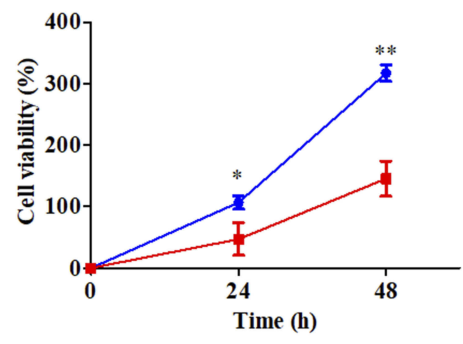

- Control

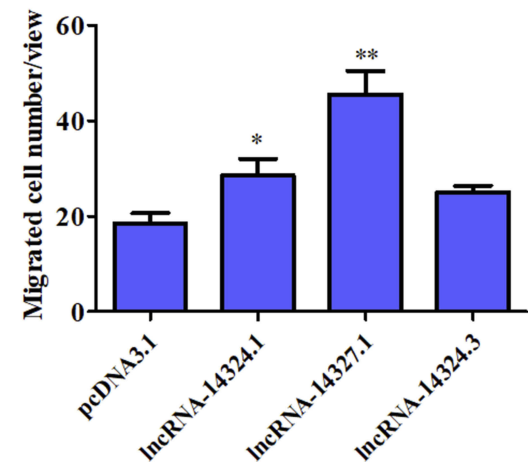

Figure 2 The role of three candidate IncRNAs on endometrial carcinoma cells. (A) qPCR analysis was performed to detect the expression of IncRNA-I4324.I, IncRNA14327. I and IncRNA-14372.3 in endometrial carcinoma HEC-IA cells transfected with pcDNA3.I, IncRNA-I4324.I, IncRNA-I4327.I or IncRNA-I4372.3-overexpression plasmids. (B) Endometrial carcinoma HEC-IA cells were transfected with pcDNA3.I, IncRNA-I4324.I, IncRNA-I4327.I or IncRNA-I4372.3-overexpression plasmids for $24 \mathrm{~h}$. Then, the cells were analyzed by Western blot for the detection of Kca3.I expression. GAPDH was used as a loading control. Quantitative analysis of Kca3.I expression was shown in the right panel. The data were derived from three independent experiments. (C) Effects of IncRNA-I4324.I, IncRNA-I4327.I, IncRNA-I4372.3 and pcDNA3.I on the proliferation of endometrial carcinoma cells were determined by MTT assay. (D)The effects of IncRNA-I4324.I, IncRNA-I4327.I, IncRNA-I4372.3 and pcDNA3.I transfection on cell migration were detected by transwell assay for $24 \mathrm{~h}$. Quantitative analysis of endometrial carcinoma cell migration shown in the right. The data are presented as the mean $\pm \mathrm{SD}$ of three independent experiments. $* \mathrm{P}<0.05$ vs control; $* * \mathrm{P}<0.01$ vs control. 
Table 2 The Sequence of Primers

\begin{tabular}{|c|c|}
\hline MSTRG. I4327.I-forward & 5'-CTGTGAATAGCCACTGCACTCCA-3' \\
\hline MSTRG. I 4327.I-reverse & 5'-GACCTGCTCCGTTTCCGACC-3' \\
\hline MSTRG.I4324.I -forward & 5'-TTTTGACCTGCTCCGTTTCC-3' \\
\hline MSTRG. I4324.I -reverse & 5'-TCGGGTGTCCGCACTAAGTT-3' \\
\hline MSTRG. I 4327.3-forward & 5'-GGAAACGGAGCAGGTCAAAA-3' \\
\hline MSTRG. 14327.3-reverse & 5'-CAAAAGAGGCAGGGTCTCACTAT-3' \\
\hline$\beta$-actin-forward & 5'-AGCACAGAGCCTCGCCTTTGC-3' \\
\hline$\beta$-actin-reverse & 5'-CTGTAGCCGCGCTCGGTGAG-3' \\
\hline
\end{tabular}

\section{The IncRNA- |4327.I Promotes Endometrial Carcinoma Cell Migration and Invasion}

Next, we detected the function of IncRNA-14327.1 in cell behavior in HEC-1A cells with stable expression of IncRNA14327.1. As shown in Figure 4A and B, stable expression of lncRNA-14327 significantly increased cells migration and invasion abilities compared to the control group.

Subsequently, the results of the MTT assay proved that the stable expression of IncRNA-14327.1 significantly inhibited cell proliferation, which was consistent with the result from the experiments with transient transfection
(Figure 4C). Inhibition of tumor cell growth is frequently related to cell cycle arrest. Therefore, we investigated the influence of lncRNA-14327.1 stable expression on the cell cycle distribution in endometrial carcinoma cell lines by Flow cytometry. The results showed that stable expression of lncRNA-14327 reduced cell cycle distribution in the S/ $M$ fraction and no significant difference in $\mathrm{G} 0 / \mathrm{G} 1$ and $\mathrm{G} 2 /$ $M$ (Figure 4D). Combined results suggested that stable expression of lncRNA-14327 promoted endometrial carcinoma cell migration and invasion, but suppressed cell proliferation by inducing cell cycle.

\section{The Couple of IncRNA-I4327.I and Kca3.I Promoted Endometrial Carcinoma Cells Migration and Invasion by Inducing EMT}

To define the role of IncRNA-14327.1 and Kca3.1 in the progression of cell metastasis in endometrial carcinoma, we treated the lenti-14327.1 HEC-1A cells with siRNA of Kca3.1. Firstly, lenti-14327.1 HEC-1A cells were associated with the decrease of the epithelium marker E-cadherin and TIMP3, an inhibitor of metalloproteinases, but the increase of
A

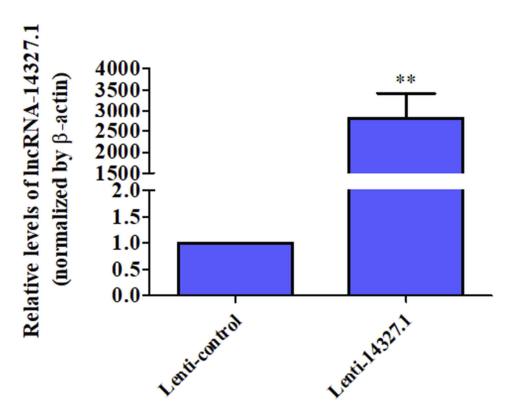

C

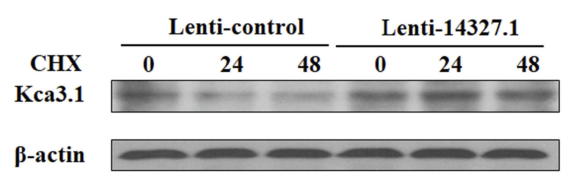

B

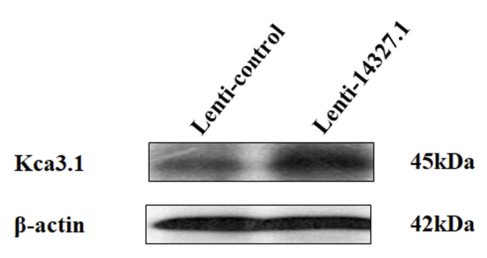

$45 \mathrm{kDa}$

$42 \mathrm{kDa}$

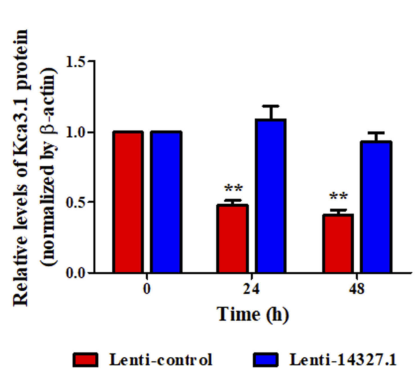

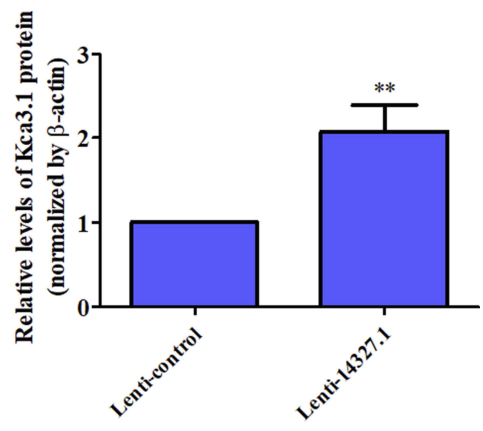

D

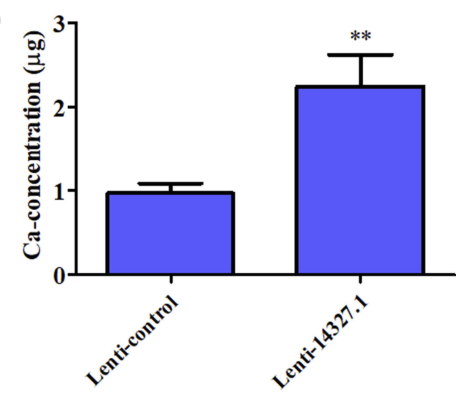

Figure 3 The IncRNA-I4327.I stabilizes Kca3.I protein to increase the intracellular concentration of Ca ${ }^{2+}$ in Lenti-I4327.I HEC-IA cells. (A) The levels of IncRNA14327.I in the lenti-14327.I group are significantly higher than those in the lenti-control group determined by qPCR assay. The data are presented as the mean \pm SD of three independent experiments. ${ }^{*} \mathrm{P}<0.0 \mathrm{I}$. (B) The protein levels of $\mathrm{Kca3}$.I in the lenti-I4327.I group are significantly higher than those in the lenti-control group determined by Western blot assay. The quantitative analysis of $\mathrm{Kca} 3 . \mathrm{I}$ is shown in the right panel. The data are presented as the mean $\pm \mathrm{SD}$ of three independent experiments. $* * \mathrm{P}<0.0 \mathrm{I}$. (C) $\mathrm{CHX}$ pulse-chase experiment demonstrating the degradation of Kca3.I protein in Lenti-control and Lenti-I4327.I HEC-IA cells. Western blot data are quantified in the right. Data are presented as mean \pm SD. $* * P<0.01$. (D) The intracellular concentration of $\mathrm{Ca}^{2+}$ in the lenti- 14327.1 group was significantly higher than those in the lenticontrol group. ${ }^{* * P}<0.01$. 

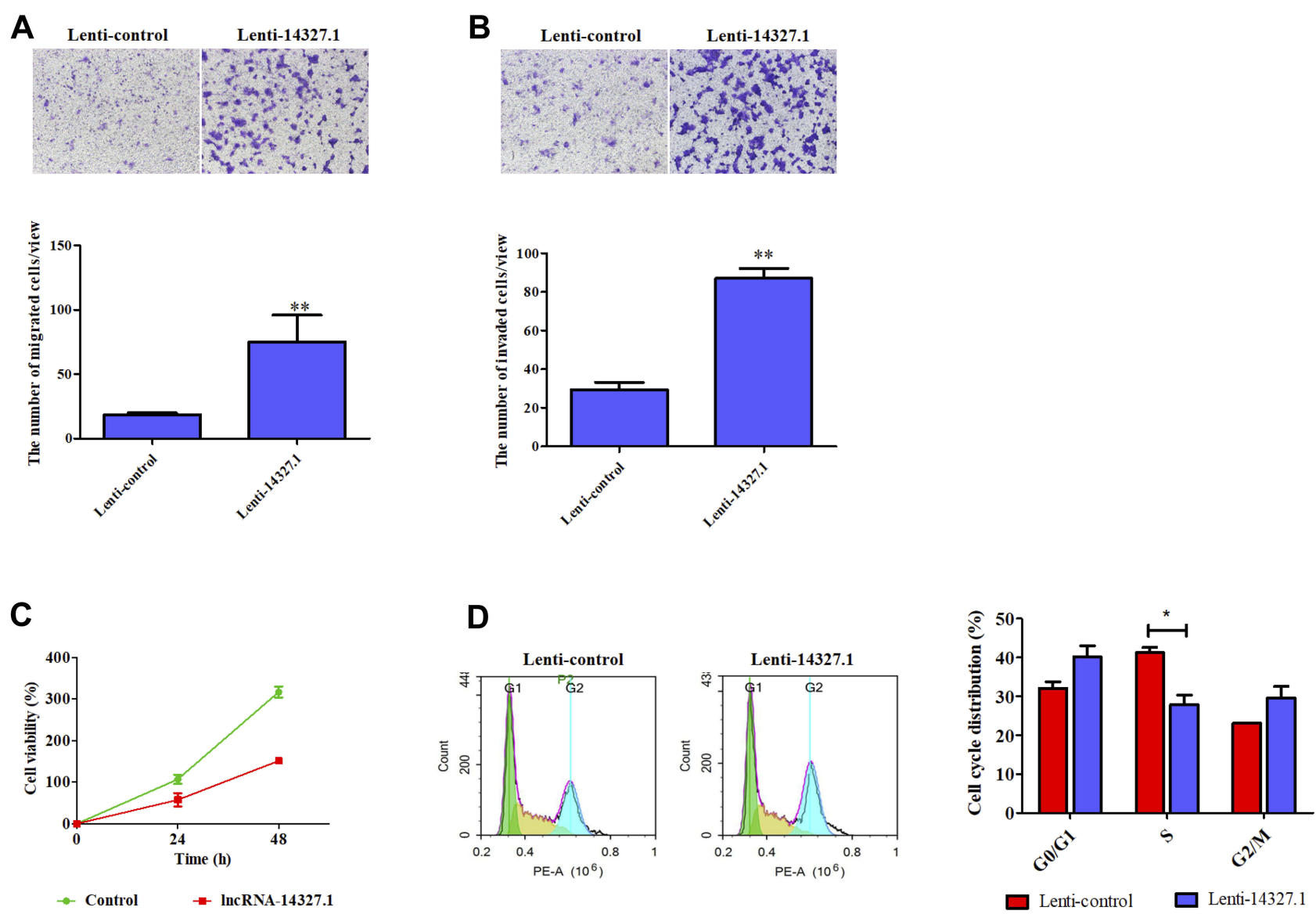

Figure 4 The IncRNA-14327.I promotes endometrial carcinoma cell migration and invasion but suppresses cell proliferation and cell cycle. (A) The IncRNA-I4327.I increased endometrial carcinoma cell migration, as measured by transwell assay. Quantitative analysis of endometrial carcinoma cell migration is shown in the below of A. The data are presented as the mean \pm SD of three independent experiments. ${ }^{*} * \mathrm{P}<0.01$. (B) The IncRNA-I4327.I strengthens endometrial carcinoma cell invasion, as measured by transwell assay precoated with matrigel. Quantitative analysis of endometrial carcinoma cell invasion is shown in the below of $B$. The data are presented as the mean \pm SD of three independent experiments. ${ }^{* * P}<0.01$. (C) The IncRNA-14327.I inhibits endometrial carcinoma cell proliferation, as measured by MTT assay. (D) Flow cytometry analysis shows a significant decrease in the number of cells in the S phase in Lenti-14327.I cells. The data are presented as the mean \pm SD of three independent experiments. ${ }^{*} \mathrm{P}<0.05$.

mesenchymal markers N-cadherin compared with those observed in the lenti-control group (Figure 5A). As shown in Figure 5B, Kca3.1 was effectively down-regulated in Kca3.1-siRNA-2 transfected cells. Next, to determine whether lncRNA-14327.1 induced endometrial carcinoma cells EMT, cell migration and invasion via coupling with Kca3.1, we made use of a Kca3.1 silence model. As shown in Figure 5C and D, we found that inhibition of Kca3.1 not only significantly decreased cell migration and invasion in the lenti-control group but also attenuated the pro-effects of lncRNA-14327.1 on cell migration and invasion. In addition, the silence of Kca3.1 inhibited the expression of two classical mesenchymal cell markers (N-cadherin) in lenti-14327.1 cells, whereas the epithelial cell marker E-cadherin and metalloproteinases inhibitor TIMP3 was elevated (Figure 5E). Taken together, all these results suggested that the lncRNA-14327.1 promoted the migration and invasion potential of endometrial carcinoma cells by upregulating Kca3.1 at least partially.

\section{Discussion}

The data from TCGA dataset revealed that the expression of Kca3.1 mRNA was upregulated in endometrial carcinoma tissues compared to adjacent noncancerous tissues and positively associated with the tumor stages. Our data from immunohistochemistry analysis also showed that the expression of Kca3.1 protein was higher expressed in endometrial carcinoma tissues compared to adjacent noncancerous tissues. Although the roles of Kca3.1 in several cancer types have been documented, the regulation mechanism for Kca3.1 expression in endometrial carcinoma remains to be illustrated, especially the role of long non-coding RNA.

The discovery of IncRNA, do not exhibit proteincoding potential, was a breakthrough in regulating the 

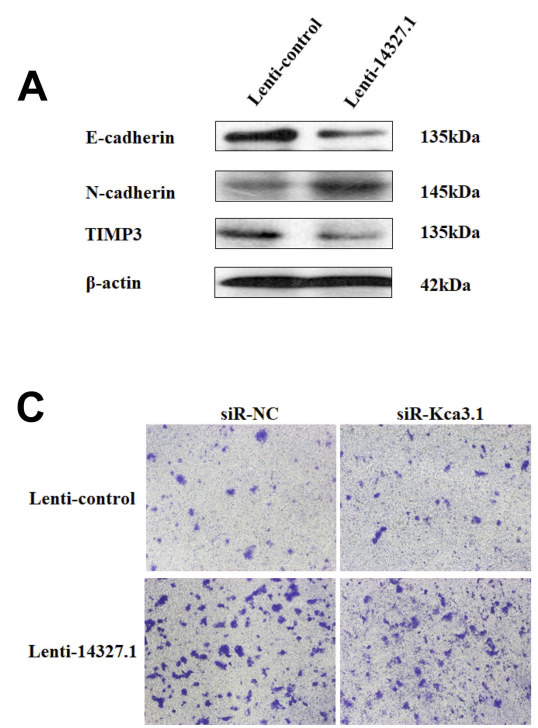

D

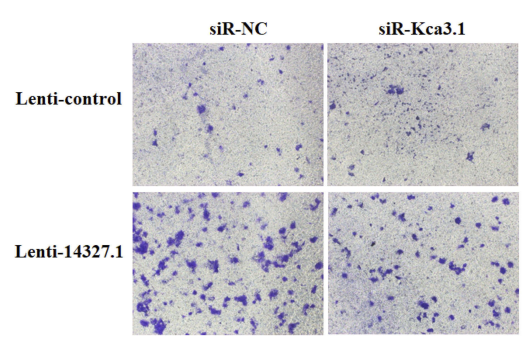

B

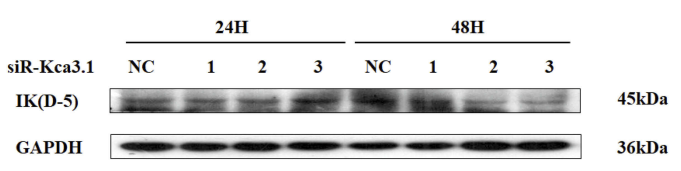

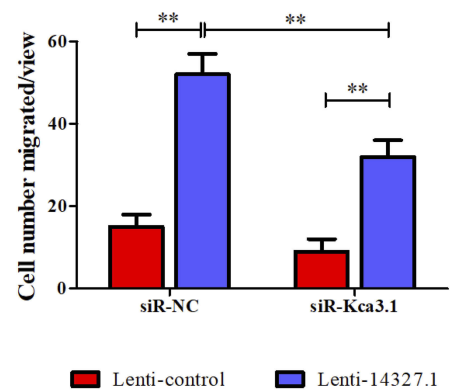

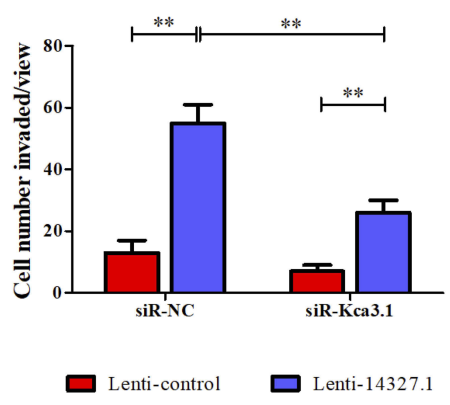

E

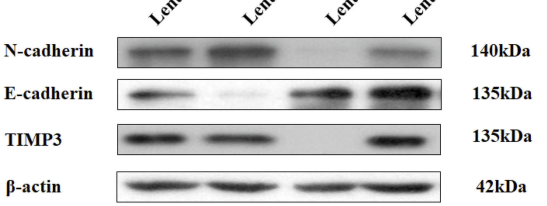

Figure 5 The couple of IncRNA-I4327.I and Kca3.I promotes endometrial carcinoma cells migration and invasion by promoting EMT. (A) Western blot analyzed the makers of EMT between Lenti-control and Lenti-I4327.I. (B) The endometrial carcinoma cells were transfected with siRNA-negative control or siRNA-Kca3.I-I, siRNAKca3.I-2, siRNA-Kca3.I-3 for $24 \mathrm{~h}$ or $48 \mathrm{~h}$. Then, the cells were analyzed by Western blot for the detection of Kca3.I expression. GAPDH was used as a loading control. (C) The abilities of cell migration induced by Lenti-I4327.I are significantly attenuated after the transfection of siRNA-Kca3.I-2 in endometrial carcinoma cells, as measured by transwell assay. The data are presented as the mean \pm SD of three independent experiments. **P $<0.01$. Error bars indicate mean \pm SD. (D) The abilities of cell invasion induced by Lenti-14327.I are significantly attenuated after the transfection of siRNA-Kca3.I-2 in endometrial carcinoma cells, as measured by transwell assay precoated with matrigel. The data are presented as the mean \pm SD of three independent experiments. **P $<0.0$ I. Error bars indicate mean \pm SD. (E) Western blot analyzed the makers of EMT in the Lenti-control and Lenti-14327.I transfection with siRNA-negative control and siRNA-Kca3.I-2, respectively. $\boldsymbol{\beta}$-actin was used as a loading control.

expression of eukaryotic genome and inducing the anomaly growth and metastasis of cancer. ${ }^{27,28}$ Operation of the expression of IncRNA could affect the cell migration and invasion, cell proliferation, cell cycle and so on of cell behavior in various cancers. ${ }^{29}$ First of all, we verified three lncRNAs, including lncRNA-14327.1, lncRNA14324.1 and lncRNA-14327.3 might directly interact with Kca3.1by RNA immunoprecipitation seq assay and PCR validation. The overexpression of all three lncRNAs could significantly promote the expression of Kca3.1 and the cell migration of HEC-1A cells, but with the cell proliferation inhibited. Furthermore, it seemed that lncRNA-14327.1 was the most efficient one. Thus, we speculated lncRNA-14327.1 might act as a molecular couple of Kca3.1 and thereby regulated Kca3.1 function.
For further elucidation for the underlying mechanism, HEC-1A cell line with stable expression of lncRNA14327 and its control cell line were constructed with lentivirus.

Our results showed that stably high expression of lncRNA-14327.1 could effectively induce endometrial carcinoma cell migration and invasion with Kca3.1 upregulated. Moreover, knockdown of Kca3.1 could partially reverse the biological response to the overexpression of lncRNA-14327.1. Therefore, our data indicated lncRNA14327.1 might promote endometrial carcinoma progression through targeting Kca3.1. Additionally, the stability of Kca3.1 protein was found to be upregulated more than twofold after overexpression of lncRNA-14327.1 in a CHX pulse-chase assay. Noticeably, a remarkable 
enhancement in the concentration of intracellular concentration of $\mathrm{Ca}^{2+}$ in the lenti-14327.1 group was observed compared with those in the lenti-control group. Overall, these data clearly demonstrated that lncRNA-14327.1 could stabilize Kca3.1 to increase the intracellular concentration of $\mathrm{Ca}^{2+}$.

One of several steps involved in metastasis is epithelialmesenchymal transition (EMT). ${ }^{30}$ As shown in Figure 5, we found that downregulation of Kca3.1 inhibited the expression of classical mesenchymal cell markers N-cadherin in lenti14327.1 cells, whereas the epithelial cell marker E-cadherin and metalloproteinases inhibitor TIMP3 was elevated. Combined results suggested that the couple of lncRNA14327.1 and Kca3.1 might induce endometrial carcinoma cells metastasis by promoting the EMT.

\section{Conclusion}

In conclusion, our findings suggest that lncRNA-14327.1 functions as a novel oncogene to regulate the cell migration and invasion of endometrial carcinoma cells by stabilizing the Kca3.1protein and subsequently activating the EMT. Therefore, the lncRNA-14327.1/Kca3.1 axis may be a novel molecular therapeutic target for the treatment of endometrial carcinoma, especially the metastatic one.

\section{Ethics Approval and Informed Consent}

All procedures performed in studies involving human participants were in accordance with the ethical standards of the Institutional Ethics Committee of the Zhejiang Cancer Hospital (Zhejiang, China) and with the 1964 Helsinki Declaration and its later amendments or comparable ethical standards.

\section{Data Sharing Statement}

All data for this study has been presented.

\section{Author Contributions}

All authors contributed to data analysis, drafting and revising the article, gave final approval of the version to be published, and agree to be accountable for all aspects of the work.

\section{Funding}

This work was supported by the National Natural Science Foundation of China (No.81602267) and Zhejiang Medical and Health Science and Technology Plan Project of China (No.2019ZH001).

\section{Disclosure}

We declare that all authors have no conflicts of interest.

\section{References}

1. Torre LA, Bray F, Siegel RL, Ferlay J, Lortet-Tieulent J, Jemal A. Global cancer statistics, 2012. CA Cancer J Clin. 2015;65(2):87-108. doi:10.3322/caac. 21262

2. Chen W, Zheng R, Baade PD, et al. Cancer statistics in China, 2015. CA Cancer J Clin. 2016;66(2):115-132. doi:10.3322/caac.21338

3. Siegel RL, Miller KD, Jemal A. Cancer statistics, 2018. CA Cancer J Clin. 2018;68(1):7-30. doi:10.3322/caac.21442

4. Cancer Genome Atlas Research N, Kandoth C, Schultz N, et al. Integrated genomic characterization of endometrial carcinoma. Nature. 2013;497(7447):67-73. doi:10.1038/nature12113

5. Nie D, Yang E, Li Z. Pretreatment thrombocytosis predict poor prognosis in patients with endometrial carcinoma: a systematic review and meta-analysis. BMC Cancer. 2019;19(1):73. doi:10.1186/s12885-0185264-y

6. Mohr CJ, Steudel FA, Gross D, et al. Cancer-Associated Intermediate Conductance $\mathrm{Ca}(2+)$-Activated $\mathrm{K}(+)$ Channel $\mathrm{KCa} 3.1$. Cancers. 2019;11(1):109. doi:10.3390/cancers11010109

7. Vergara C, Latorre R, Marrion NV, Adelman JP. Calcium-activated potassium channels. Curr Opin Neurobiol. 1998;8(3):321-329. doi:10.1016/S0959-4388(98)80056-1

8. D'Alessandro G, Monaco L, Catacuzzeno L, et al. Radiation increases functional $\mathrm{KCa} 3.1$ expression and invasiveness in glioblastoma. Cancers. 2019;11(3):279. doi:10.3390/cancers11030279

9. Bonito B, Sauter DR, Schwab A, Djamgoz MB, Novak I. KCa3.1 (IK) modulates pancreatic cancer cell migration, invasion and proliferation: anomalous effects on TRAM-34. Pflugers Arch. 2016;468 (11-12):1865-1875. doi:10.1007/s00424-016-1891-9

10. Rabjerg M, Olivan-Viguera A, Hansen LK, et al. High expression of $\mathrm{KCa} 3.1$ in patients with clear cell renal carcinoma predicts high metastatic risk and poor survival. PLoS One. 2015;10(4):e0122992. doi:10.1371/journal.pone. 0122992

11. Robles-Martinez L, Garay E, Martel-Gallegos MG, et al. Kca3.1 activation via $\mathrm{P} 2 \mathrm{y} 2$ purinergic receptors promotes human ovarian cancer cell (Skov-3) migration. Sci Rep. 2017;7(1):4340. doi:10.1038/s41598-017-04292-6

12. Pillozzi S, D'Amico M, Bartoli G, et al. The combined activation of $\mathrm{KCa} 3.1$ and inhibition of Kv11.1/hERG1 currents contribute to overcome cisplatin resistance in colorectal cancer cells. $\mathrm{Br} \mathrm{J}$ Cancer. 2018;118(2):200-212. doi:10.1038/bjc.2017.392

13. De Marchi U, Sassi N, Fioretti B, et al. Intermediate conductance $\mathrm{Ca} 2+$-activated potassium channel $(\mathrm{KCa} 3.1)$ in the inner mitochondrial membrane of human colon cancer cells. Cell Calcium. 2009;45 (5):509-516. doi:10.1016/j.ceca.2009.03.014

14. Faouzi M, Hague F, Geerts D, et al. Functional cooperation between KCa3.1 and TRPC1 channels in human breast cancer: role in cell proliferation and patient prognosis. Oncotarget. 2016;7 (24):36419-36435. doi:10.18632/oncotarget.v7i24

15. Bulk E, Kramko N, Liashkovich I, et al. KCa3.1 channel inhibition leads to an ICAM-1 dependent increase of cell-cell adhesion between A549 lung cancer and HMEC-1 endothelial cells. Oncotarget. 2017;8 (68):112268-112282. doi:10.18632/oncotarget.22735

16. Ruggieri P, Mangino G, Fioretti B, et al. The inhibition of $\mathrm{KCa} 3.1$ channels activity reduces cell motility in glioblastoma derived cancer stem cells. PLoS One. 2012;7(10):e47825. doi:10.1371/journal. pone. 0047825 
17. Mercer TR, Dinger ME, Mattick JS. Long non-coding RNAs: insights into functions. Nat Rev Genet. 2009;10(3):155-159. doi:10.1038/nrg2521

18. Engreitz JM, Ollikainen N, Guttman M. Long non-coding RNAs: spatial amplifiers that control nuclear structure and gene expression. Nat Rev Mol Cell Biol. 2016;17(12):756-770. doi:10.1038/nrm.2016.126

19. Aird J, Baird AM, Lim MCJ, McDermott R, Finn SP, Gray SG. Carcinogenesis in prostate cancer: the role of long non-coding RNAs. Noncoding RNA Res. 2018;3(1):29-38. doi:10.1016/j. ncrna.2018.01.001

20. Batista PJ, Chang HY. Long noncoding RNAs: cellular address codes in development and disease. Cell. 2013;152(6):1298-1307. doi:10.1016/j.cell.2013.02.012

21. Yang F, Wen S, Zhang Y, et al. Identifying potential metastasisrelated long non-coding RNAs, microRNAs, and message RNAs in the esophageal squamous cell carcinoma. J Cell Biochem. 2019.

22. Schmitt AM, Chang HY. Long noncoding RNAs in cancer pathways. Cancer Cell. 2016;29(4):452-463. doi:10.1016/j.ccell. 2016.03.010

23. Liu H, Zhang Z, Xiong W, et al. Long non-coding RNA MALAT1 mediates hypoxia-induced pro-survival autophagy of endometrial stromal cells in endometriosis. J Cell Mol Med. 2019;23 (1):439-452. doi:10.1111/jcmm.2019.23.issue-1
24. Li Q, Zhang C, Chen R, et al. Disrupting MALAT1/miR-200c sponge decreases invasion and migration in endometrioid endometrial carcinoma. Cancer Lett. 2016;383(1):28-40. doi:10.1016/j.canlet.2016.09.019

25. Chi S, Liu Y, Zhou X, et al. Knockdown of long non-coding HOTAIR enhances the sensitivity to progesterone in endometrial cancer by epigenetic regulation of progesterone receptor isoform B. Cancer Chemother Pharmacol. 2019;83(2):277-287. doi:10.1007/s00280-018-3727-0

26. Zhang L, Wang DL, Yu P. LncRNA H19 regulates the expression of its target gene HOXA10 in endometrial carcinoma through competing with miR-612. Eur Rev Med Pharmacol Sci. 2018;22 (15):4820-4827. doi:10.26355/eurrev_201808_15617

27. Serviss JT, Johnsson P, Grander D. An emerging role for long non-coding RNAs in cancer metastasis. Front Genet. 2014;5:234. doi:10.3389/fgene.2014.00234

28. Zhang DM, Lin ZY, Yang ZH, et al. IncRNA H19 promotes tongue squamous cell carcinoma progression through beta-catenin/ GSK3beta/EMT signaling via association with EZH2. Am J Transl Res. 2017;9(7):3474-3486.

29. Chatterjee M, Sengupta S. Emerging roles of long non-coding RNAs in cancer. $J$ Biosci. 2019;44(1):22. doi:10.1007/s12038-018-9820-Z

30. Thiery JP, Acloque H, Huang RY, Nieto MA. Epithelialmesenchymal transitions in development and disease. Cell. 2009;139(5):871-890. doi:10.1016/j.cell.2009.11.007
OncoTargets and Therapy

\section{Publish your work in this journal}

OncoTargets and Therapy is an international, peer-reviewed, open access journal focusing on the pathological basis of all cancers, potential targets for therapy and treatment protocols employed to improve the management of cancer patients. The journal also focuses on the impact of management programs and new therapeutic agents and protocols on patient perspectives such as quality of life, adherence and satisfaction. The manuscript management system is completely online and includes a very quick and fair peer-review system, which is all easy to use. Visit http://www.dovepress.com/ testimonials.php to read real quotes from published authors. 